\title{
FRAIL OLD PATIENTS AS A TARGET POPULATION FOR CANCER TRIALS
}

\author{
J.-P. MICHEL¹, S. PAUTEX'1, M. AAPRO², G. ZULIAN \\ 1. Department of Rehabilitation and Geriatrics Medical School and University Hospitals; 2. Clinics of Genolier . Geneva - Switzerland
}

\begin{abstract}
The recent distinction between co-morbidity and multi-morbidity well stresses the difficulty of managing old patients with cancer whose complexity is not captured by a list of diagnoses or biological burden alone. The most adequate answer found by oncologists and geriatricians was to work together for better evaluating the physiological age and body reserve of the patient. The gold standard tool to assess old patient with cancer is named Comprehensive Geriatric Assessment. Its systematic application needs geriatric competences and time. In this context, a great number of cancer patients are considered as "frail" because they have reduced available physiological reserves. They might not withstand stress when challenged. Oncologists and geriatricians have imagined an innovative process to change the screening procedure of these patients, determine the prognosis, adapt the treatment strategy, to increase the patient's survival and his/her quality of life. The internet website "www.clinicaltrials.com" only lists 8 studies focused on frail elders with cancer. Six of them are focused on specific cancers or specific treatments, one was applied to all kind of cancers and the last was an opinion overview from oncologists and geriatricians. The selection criteria of frail patients are very diverse and probably include cancer patients who are not comparable. It is now time to try to identify new practical, reliable and accurate tools to facilitate the inclusion of the same kind of patients suffering from the same kind of cancer to be able to give more appropriate care and at the same time to constitute a valuable data base. Existing tools are reviewed and analyzed.
\end{abstract}

Ageing of the population is accompanied by an important health shift. On the one hand, there is a decreased prevalence of senior adults presenting with one single disease, so-called "primary" or "index" disease, and on the other hand, there is an increased prevalence of co-occurring diseases in the same individual (1).

To better address these issues and their consequences, the NIA taskforce co-existing health conditions recently proposed new definitions (2). The term "co-morbidity" corresponds to one primary or index disease associated with specific 2 or multiple concurrent conditions, while the term "multimorbidity" corresponds to the sum of conditions without a specific primary or index disease (2). Applied to cancer patients, these definitions stress the management challenge of oncologists dealing with aged patients whose complexity is no more captured by lists of diagnoses or biological burden alone $(3,4,5)$. The co-existing health conditions of many senior cancer patients require tailoring the screening procedure, to adapt the treatment options and to modify the prognosis evaluation $(3,4,5)$.

The most publicized scoring system for senior cancer patients is the comprehensive geriatric assessment (CGA) (6). It consists of a multidimensional data-search and analytic process of patient characteristics aiming to an individualized intervention-plan. CGA was proven to allow comprehensive identification of the patients' health problems and to facilitate the choice of the most adequate treatments $(7,8)$. However, neither is the survival quantity enhanced nor is its quality improved by CGA (8). A combination of CGA and of supportive measure during follow-up management is therefore an attractive option (7).

In this context, the emergence of the concept of frailty is likely to further complicate the already existing complexity of senior of cancer patients, whose clinical features are quite similar. Frail subjects are anorexic, weak, exhausted, and inactive with impaired homeostatic reserves and reduced capacity to withstand any kind of stress (9). The health consequences of frailty modify cancer management with a negative impact on prognosis. Recurrent falls, trauma, polypharmacy, repeated hospital admissions may lead to crossinfections, disability and death (10).

The challenge of not missing frailty features in senior cancer patients explains the request to better identify this subtle condition in cancer trials.

\section{Randomized controlled trials focused on frail elders with cancer}

The website www.clinicaltrials.gov gathers and updates all randomized controlled studies performed throughout the world (11). Forty are dedicated to frail elders with only 8 devoted to frail cancer elders. Among these, six are disease or treatment specific, one includes all kind of cancers and one is an ongoing opinion analysis. Main issue of these studies is to determine how frail senior cancer patients are assessed.

\section{Randomized controlled studies of frail elders with cancer}

Inclusion criteria in these studies illustrate the diversity of opinions about the frailty syndrome: i) age 60 to 70 without comorbid illness ii) patients with decreased cardiac ejection fraction or pulmonary dysfunction or elevated liver tests or hepatitis $\mathrm{C}$ infection or poor performance status iii) $65+$ female, post menopausal status and one of the following characteristics of frailty: over 85 years of age, history of 3 falls in the past 6 months, more than 3 co-morbidities, mild dementia (must be oriented in time, space and location) and at least one ADL inability (11). The criteria used to define "frail old cancer 
patients" have thus not reached the stage of a consensual operational definition.

In a study about 99 frail cancer patients who receive inand/or out-patients specialized geriatric care after stabilisation of their diseases, at least 2 of the following conditions defined frailty: previous stroke, falls, hospital admission 3 months before inclusion, 1 or 2 ADL inabilities, difficulty ambulating, prolonged bed rest, malnutrition, incontinence, dementia and depression. Such criteria are very broad and may include other geriatric patients than the frail. With no surprise, the results of the study demonstrated better pain control and better mental health scores after 1 year in in-patients receiving specialized geriatric care (12) .

\section{Randomized controlled surveys on opinion analysis}

The unique on-going survey on this topic concerns views and attitudes of oncologists $(\mathrm{N}=150)$ and general practitioners, internists and geriatricians $(\mathrm{N}=150)$ on the use of chemotherapy/hormone therapy in older and/or frail women with breast cancer. Inclusion criteria for doctors include more than 2 years in practice, more than 25 post menopausal breast cancer patients on adjuvant hormonal therapy under care and not being linked to any advertising, market research or drug company. The main goals of the survey are i) to evaluate the impact on care management and treatment recommendations of age, health and daily functioning of patients and ii) to determine if any consensual attitudes exist between medical doctors with different training (11). The results of this survey will be of importance to define better inclusion criteria of frail senior cancer patients and to avoid age discrimination.

Despite many ongoing difficulties in the definition of frailty, it is encouraging to see oncologists taking into account the physiological age of patients which is the reflection of a normal but sometimes abnormal accelerated loss of body reserves (13). It is though true that CGA is time consuming and that too few geriatricians are available to help in caring for old and frail cancer patients.

\section{Searching new assessment tools in frail cancer patients}

A recent review of all geriatric assessment tools for cancer patients underlines once again the value of CGA. Its limited use in daily clinical practice is highlighted with the need to identify more convenient functional scales (6). A comparison between CGA and different types of frailty assessment scales is thus needed.

\section{Very few tools were already tested in cancer patients}

The Cumulative Illness Rating Scale - Geriatric (CIRS-g) was used in breast (14), lung (15) and rectal (16) cancer patients to determine the impact of co-morbidity on the overall survival. In each of the above mentioned study, co-morbidity score increases with age. However, age, co-morbidity and weight loss (17) or disease severity (18) are also independent prognostic factors of overall survival

The Karnosky Performance Status (KPS) is of interest in young patients but it has not been validated in senior adult cancer patients (6). A retrospective analysis of survival factors in 30 patients aged 70 and older with grade 3 and 4 malignant gliomas showed that KPS is the only significant prognostic factor in multivariate analysis (19). Prospective studies of KPS in old cancer patients are thus needed.

\section{The Vulnerable Elders Scale (VES-13)}

It is a 5-minutes self-administrated scale including 4 categories of items corresponding to age, self rated health, physical disabilities $(\mathrm{N}=6)$ and functional disabilities $(\mathrm{N}=5)$ (20). Neither co-morbidity data nor mental/cognitive evaluation is part of the evaluation.

VES-13 was compared to CGA in a pilot study of 50 prostate cancer patients ( 70 to 82 years) treated with androgen ablation. The Pearson correlation coefficient testified the reliability of VES-13 at 0.92 . However, when excluding the age criteria, reliability of VES-13 went down to 0.52 for self-rated health, 0.60 for self rated physical ability and 0.70 for self rated functional ability (21).

The Mini Nutrional Assessment (MNA) short form was part of a CGA including also MMSE, get up and go test, bADL, iADL, GDS and CIRS-G to evaluate 364 cancer patients (median age: 77.5 y., sex ratio $\mathrm{H} / \mathrm{F} 1.43$ ) accrued in 12 French centres. They were suffering of different cancers: 110 lymphomas, 101 colon, 37 stomach, 37 lung, 23 pancreas, 20 prostate, 18 bladder, 14 ovary cancers and 4 from unknown location and type. Sixty six percent of the cancer patients had advanced tumours. The early death predicted by logistic regression was linked to the MNA short form $(\mathrm{O} . \mathrm{R} .=4.5[1.75$ -11.6]) and the advanced disease (O.R. = 4.2 [1.70 -10.3]) (22). These encouraging results must be confirmed by prospective studies keeping in mind that short survival is not a frailty feature but the most dramatic consequence of the frailty syndrome.

The Abbreviated CGA (aCGA) corresponds to a simplified form of CGA including 15 selected items from the main geriatric scales: 6 from the MMSE, 4 from the GDS, 3 from the bADL and 4 from the iADL. The global score determines the benefit for the patient to get an entire CGA (eCGA). The aCGA was retrospectively used for the evaluation of 500 charts of old cancer patients. The aCGA / eCGA correlations ranged from 0.84 to 0.96 , testifying that aCGA can be helpful in screening for patients who would benefit from an eCGA (23). As previously mentioned, it is not yet known whether aCGA has an impact on treatment decision, choice and tolerance as well as on survival.

\section{Other potential screening tools not yet tested in frailty cancer patients}

Numerous other tools have been used to assess the frailty status of non-cancer old persons. It seems useful to mention some of them focusing on gait ability.

\section{The one leg standing balance test}

This test applied in a cross-sectional study in an urban community dwelling population $(\mathrm{N}=512$, m.a. $=73 \mathrm{y} \pm 7.0$, 


\section{THE JOURNAL OF NUTRITION, HEALTH \& AGING@}

$71.4 \%$ women) showed that $24.7 \%$ had abnormal one leg standing balance. In $60.6 \%$, abnormalities are linked to an iADL deficit. In addition, abnormal one leg standing balance is significantly linked to age over 71 years $($ O.R. $=5.11[\mathrm{CI}=$ $1.99-13.10])$, iADL deficit (transports) $(\mathrm{O} . \mathrm{R} .=3.61[\mathrm{CI}=$ $1.15-11.40]$ ) and poor health status (O.R. $=2.67$ [CI $=1.35-$ 5.27]) 24 .

\section{The gait speed}

Four hundred twenty four volunteers aged 70-89 years were randomly assigned to two interventions: physical activity or educational programme for a planned minimum of 12 months. They were assessed by 4 different techniques: short physical performance battery, self-reported disability scale, 4 meters walks (less than 10 seconds) and 400 meters walk (less than 15 minutes). The ability to walk 400 meters as a dichotomous outcome appears as the most interesting measure to appreciate the results of the intervention programme (25).

\section{The gait velocity}

Gait velocity was tested in 102 well functioning participants (m.a. $=79.6 \pm 4$ years) and then followed-up during 24 months. Low gait velocity $(<0.7 \mathrm{~m} / \mathrm{s})$ measured on a 8 to 10 meters walk appeared to be a good predictor of requirement of a care giver (RR: 9.5 [1.3 - 2.5]), hospitalization (RR: 5.9 [1.9 - 8.5]) and new falls (RR: 5.4 [12.0 - 4.3]) (26).

\section{The gait variability}

The gait variability of 379 older adults (m.a = 79 y.) community dwelling, free of mobility disability by self report was tested using a GaitMat II (4-meter-long walkway). Then, mobility disability was self reported by telephone contact every 6 months during 54 months. The incident mobility disability reached $58.6 \%$ during the study period. Cox proportional hazard models after adjusting for gait speed, demographics, chronic conditions, prescription of medications, health status and level of physical activities showed that the initial increased in stance time variability of 0.001 second. Is linked with a $13 \%$ higher incidence of mobility disability (27).

\section{Conclusion}

The need of senior cancer patients to benefit of a comprehensive geriatric assessment including follow-up management was well demonstrated. It allows i) a better identification of the health problems of the cancer patient, including frailty-risk, ii) an easier choice of appropriate and better tolerated treatment iii) a longer survival. However the main difficulties of oncologists are linked to the availability of geriatricians performing such a long assessment. The need to find and validate short pre-treatment assessment tools for senior cancer patients appears urgent. The analysis of past and ongoing randomized controlled trials in frail patients with cancer emphasized on further and larger research.

Pre-treatment geriatric assessment i) has to be validated in patients whatever their age ii) and whatever the cancer type iii) will facilitate the choice of cancer treatments iv) will have to be followed by geriatric management and v) will allow longer and hopefully better survival. Geriatricians are now confronted to outstanding research challenges. Results of large randomized controlled trials will soon be able to predict frailty thanks to systematic evaluation with simple, easy and reliable tools.

Financial disclosure: None of the authors had any financial interest or support for this paper.

\section{References}

1. Wolff JL, Starfield B, Anderson G. Prevalence, expenditures, and complications of multiple chronic conditions in the elderly. Arch Intern Med 2002;162(20):2269-76.

2. Yancik R, Ershler W, Satariano W, Hazzard W, Cohen HJ, Ferrucci L. Report of the national institute on aging task force on comorbidity. J Gerontol A Biol Sci Med Sci 2007;62(3):275-80

3. Walter LC, Covinsky KE. Cancer screening in elderly patients: a framework for individualized decision making. Jama 2001;285(21):2750-6.

4. Yancik R, Wesley MN, Ries LA, Havlik RJ, Edwards BK, Yates JW. Effect of age and comorbidity in postmenopausal breast cancer patients aged 55 years and older. Jama 2001;285(7):885-92.

5. Bynum JP, Braunstein JB, Sharkey P, Haddad K, Wu AW. The influence of health status, age, and race on screening mammography in elderly women. Arch Intern Med 2005;165(18):2083-8

6. Gosney M. Contribution of the geriatrician to the management of cancer in older patients. Eur J Cancer 2007;43(15):2153-60.

7. Extermann M, Hurria A. Comprehensive geriatric assessment for older patients with cancer. J Clin Oncol 2007;25(14):1824-31.

8. Maas HA, Janssen-Heijnen ML, Olde Rikkert MG, Machteld Wymenga AN. Comprehensive geriatric assessment and its clinical impact in oncology. Eur J Cancer 2007:43(15):2161-9.

9. Fried LP, Tangen CM, Walston J, et al. Frailty in older adults: evidence for a phenotype. J Gerontol A Biol Sci Med Sci 2001;56(3):M146-56.

10. Lally F, Crome P. Understanding frailty. Postgrad Med J 2007;83(975):16-20.

11. National Cancer Institute. www.ClinicalTrials.gov 2007.

12. Rao AV, Demark-Wahnefried W. The older cancer survivor. Crit Rev Oncol Hematol 2006;60(2):131-43.

13. Aapro M. About geriatric oncology. Eur J Cancer 2007:43(15):2141-3.

14. Extermann M, Meyer J, McGinnis M, et al. A comprehensive geriatric intervention detects multiple problems in older breast cancer patients. Crit Rev Oncol Hematol 2004;49(1):69-75.

15. Firat S, Bousamra M, Gore E, Byhardt RW. Comorbidity and KPS are independent prognostic factors in stage I non-small-cell lung cancer. Int J Radiat Oncol Biol Phys 2002;52(4):1047-57.

16. Wedding U, Roehrig B, Klippstein A, et al. Comorbidity in patients with cancer: prevalence and severity measured by cumulative illness rating scale. Crit Rev Oncol Hematol 2007;61(3):269-76.

17. Firat S, Pleister A, Byhardt RW, Gore E. Age is independent of comorbidity influencing patient selection for combined modality therapy for treatment of stage III nonsmall cell lung cancer (NSCLC). Am J Clin Oncol 2006;29(3):252-7.

18. Firat S, Byhardt RW, Gore E. Comorbidity and Karnofksy performance score are independent prognostic factors in stage III non-small-cell lung cancer: an institutional analysis of patients treated on four RTOG studies. Radiation Therapy Oncology Group. Int J Radiat Oncol Biol Phys 2002;54(2):357-64.

19. Kurimoto M, Nagai S, Kamiyama H, et al. Prognostic factors in elderly patients with supratentorial malignant gliomas. Neurol Med Chir (Tokyo) 2007;47(12):543-9; discussion 549

20. Saliba D, Orlando M, Wenger NS, Hays RD, Rubenstein LZ. Identifying a short functional disability screen for older persons. J Gerontol A Biol Sci Med Sci 2000;55(12):M750-6.

21. Mohile SG, Bylow K, Dale W, et al. A pilot study of the vulnerable elders survey-13 compared with the comprehensive geriatric assessment for identifying disability in older patients with prostate cancer who receive androgen ablation. Cancer 2007;109(4):802-10.

22. SOUBEYRAN PL et al,. Abstract 9040, ASCO, 2007

23. Overcash JA, Beckstead J, Moody L, Extermann M, Cobb S. The abbreviated comprehensive geriatric assessment (aCGA) for use in the older cancer patient as a prescreen: scoring and interpretation. Crit Rev Oncol Hematol 2006;59(3):205-10.

24. Vellas BJ, Wayne SJ, Romero L, Baumgartner RN, Rubenstein LZ, Garry PJ. One-leg balance is an important predictor of injurious falls in older persons. J Am Geriatr Soc 1997:45(6):735-8.

25. Espeland MA, Gill TM, Guralnik J, et al. Designing clinical trials of interventions for mobility disability: results from the lifestyle interventions and independence for elders pilot (LIFE-P) trial. J Gerontol A Biol Sci Med Sci 2007;62(11):1237-43.

26. Montero-Odasso M, Schapira M, Soriano ER, et al. Gait velocity as a single predictor of adverse events in healthy seniors aged 75 years and older. J Gerontol A Biol Sci Med Sci 2005;60(10):1304-9.

27. Brach JS, Studenski SA, Perera S, VanSwearingen JM, Newman AB. Gait variability and the risk of incident mobility disability in community-dwelling older adults. J Gerontol A Biol Sci Med Sci 2007;62(9):983-8 